\title{
Mermaid Syndrome
}

\author{
Siddique Md AB' ${ }^{1}$, Saha $S^{2}$, Bose $K^{3}$, Ghorai $S^{4}$, Khan $K^{5}$
}

\begin{abstract}
We report a case of sirenomelia baby (Mermaid syndrome) born to a twenty three years old female at 37 weeks of gestation. Sirenomelia is characterized by fusion of the lower limbs with absent kidneys, absent external genitalia and other gastrointestinal defects. It results from the failure of normal vascular supply from the lower aorta in vitro, maternal Diabetes mellitus has been associated with caudal regression syndrome and sirenomelia.
\end{abstract}

Key Words: Mermaid syndrome, Sirenomelia, caudal regression.

\section{Introduction}

irenomelia, or the mermaid syndrome, is an extreme example of the caudal regression syndrome. It is found approximately $1: 100,000$ live births ${ }^{1}$ and is fatal within the first day because of complication of abnormal kidney and urinary system development. Mermaid syndrome was first described by Rocheus in 1542 and Palfyn in 1553 and called after the mythical Greek sirens ${ }^{2,3}$. This lethal anomaly is associated with a number of genitourinary and anorectal defects $^{3,4}$. The underlying visceral anomalies are usually such that the syndrome is incompatible with life yet there are a number of reported cases of surviving infants with this condition in the English literature ${ }^{5,6,7}$. We report here the first documented case of sirenomelia in Kolkata (West Bengal) and discuss our findings in relation to the present literature and related controversies of its aetiopathogenesis.

\section{The Case}

A 23 year old woman with history of no ingestion of any teratogenic agents or not having diabetes mellitus, delivered a live baby at 37 weeks of gestation with fused lower limbs. Mother was from a very poor socioeconomic group, without any antenatal checkups. No antenatal USG was available. The baby didn't cry immediately after birth and required resuscitation in the form of bag and mask ventilation. The Apgar score was 3 at 1 and same at 5 min following which the baby was shifted to neonatal intensive care unit, but died four hours after birth. After discussing with parents and as per their request not to resuscitate baby was kept under free oxygen musk. Examination of the baby revealed caudal dysgenesis having fusion of lower limbs, single leg with one foot and seven toes. There was no identifiable external genitalia and anus. X-rays revealed lower limbs
${ }^{1}$ Dr. Md Abu Bakkar Siddique, MBBS, MD (Junior Resident), ${ }^{2} \mathrm{Dr}$. Sudip Saha, Associate Professor, ${ }^{3} \mathrm{Dr}$. Kallol Bose, MBBS. MD, Assistant Professor, ${ }^{4} \mathrm{Dr}$. Sudipta Ghorai, MBBS, MD (Senior Resident), ${ }^{5}$ Dr. Kalimuddin Khan, Paediatric Medicine, MBBS. MD (Junior Resident). All from the Department of Paediatric Medicine, Chittaranjan Seva Sadan College of Obstetrics, Gynaecology and Child Health, Kolkata, West Bengal, India.

\section{Address for correspondence \\ Dr. Abu Bakkar Siddique \\ E-mail: abu89.cmc@gmail.com}

\section{How to cite}

Siddique Md AB, Saha S, Bose K, Ghorai S, Khan K. Mermaid Syndrome. J Nepal Paediatr Soc 2018;38(2):132-134.

doi:http://dx.doi.org/10.3126/jnps.v38i2.18802

This work is licensed under a Creative Commons Attribution 3.0 License. 
were fused completely into single limb and two femur and one tibia were seen.

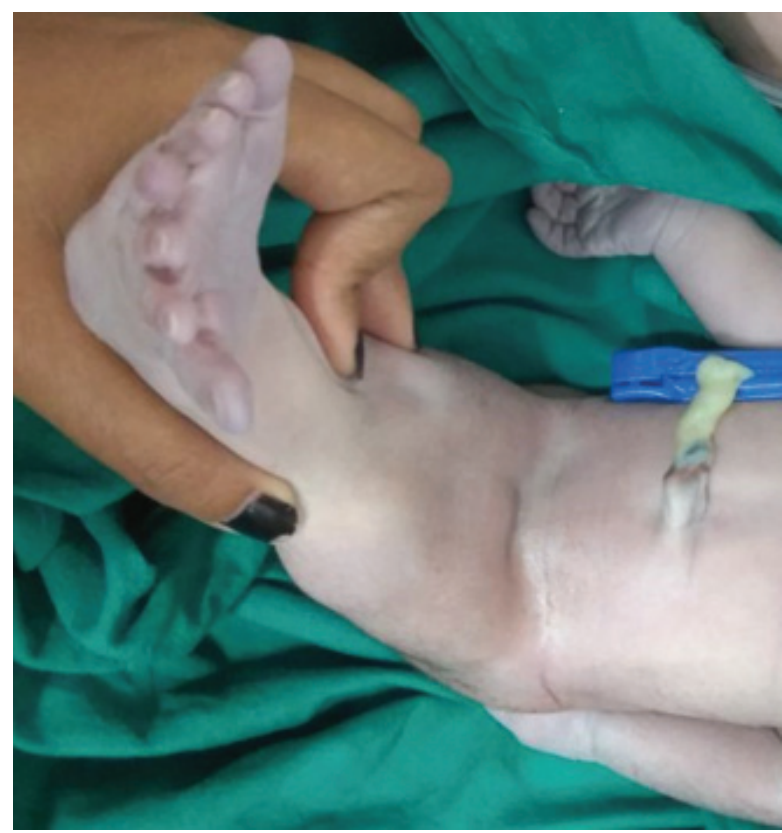

Fig 1: Mermaid baby showing no external genitalia (mobile phone photograph).

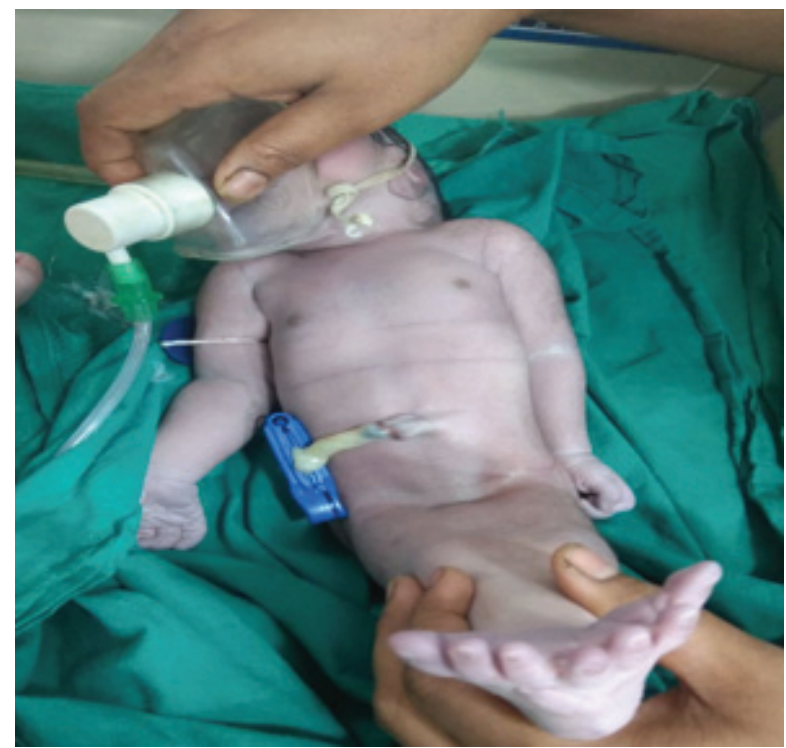

Fig 2: Mermaid baby showing fused lower limb (mobile phone photograph).

\section{Discussion}

Sirenomelia, which is also known as mermaid syndrome, is an extremely rare congenital developmental disorder characterized by anomalies of the lower spine and the lower limbs. Affected infants are born with partial or complete fusion of the legs. Additional malformations may also occur including genitourinary abnormalities, gastrointestinal abnormalities, anomalies of the lumbosacral spine and pelvis and absence or underdevelopment (agenesis) of one or both kidneys.
Affected infants may have one foot, no feet or both feet, which may be rotated externally. The tailbone is usually absent and the sacrum is partially or completely absent as well. Some sources in the medical literature classify sirenomelia as the most severe form of caudal regression syndrome, a complex developmental disorder. However, recently many researchers have indicated that sirenomelia is a similar, but distinct, disorder.

There are a wide range of physical malformations that can potentially occur with sirenomelia and the specific findings can vary greatly from one individual to another. Sirenomelia is associated with severe lifethreatening complications and is often fatal in the first years of life. However, survival beyond infancy into later childhood or young adulthood has been reported in a handful of cases.

The characteristic finding of sirenomelia is partial or complete fusion of the lower legs. The degree of severity is highly variable. Affected infants may have only one femur (the long bone of the thigh) or may have two femurs within one shaft of the skin. Affected infants may have one foot, no feet or both feet, which may be rotated so that the back of the foot is facing forward.

Infants with sirenomelia may also have abnormalities affecting the sacral and lumbar spine. In some patients, abnormal front-to-back curvature of the spine (lordosis) may occur. Affected individuals may also lack external genitalia. Absence of the spleen and/ or the gallbladder has also been reported.

Defects affecting the abdominal wall may also occur such as protrusion of a portion of the intestines through a hole near the bellybutton (omphalocele). Some individuals with sirenomelia may have a meningomyelocele, a condition in which the membranes that cover the spine and, in some cases, the spinal cord itself protrude through a defect in the spinal column. Congenital heart defects and respiratory complications such as severe underdevelopment of the lungs (pulmonary hypoplasia) can also be associated with sirenomelia.

The exact cause of sirenomelia is unknown. Researchers believe that both environmental and genetic factors may play a role in the development of the disorder. Most cases appear to occur randomly for no apparent reason (sporadically), which suggests environmental factors or a new mutation. Most likely, sirenomelia is multifactorial, which means that several different factors may play a causative role. In addition, different genetic factors may contribute to the disorder in different people (genetic heterogeneity). 
Stocker and Heifetz classified sirenomelia in seven types $^{8}$

(I) All thigh and leg bones present.

(II) Single fibula.

(III) Absent fibulae.

(IV) Partially fused femurs, fused fibulae.

(V) Partially fused femurs, absent fibulae.

(VI) Single femur, single tibia.

(VII) Single femur absent tibiae.

Several etiologic factors have been suggested:

1. Maternal diabetes is the only maternal disease known to be associated with sirenomelia. However it is more common with CRS.

2. Teratogens like retinoic acid, cadmium, cyclophosphamide have reported to cause sirenomelia in mice. However no case of sirenomelia has been observed on accidental exposure of these products. Cocaine, organic solvents of fat and appetite suppressors (diethylpropion) has been implicated in some cases of CRS in humans ${ }^{9}$.

3. Several family cases of CRS have been reported suggesting genetic predisposition

4. "Vascular steal" theory by Stevenson on the basis of dissection of the abdominal vasculature in eleven cases of sirenomelia has demonstrated a pattern of vascular abnormality ${ }^{10}$. The common feature is the presence of a single large artery, arising from high in the abdominal cavity, which assumes the function of the umbilical arteries and diverts nutrients from the caudal end of the embryo distal to the level of its origin. The steal vessel derives from the vitelline artery complex, an early embryonic vascular network that supplies the yolk sac. Arteries below the level of this steal vessel are underdeveloped and tissues dependent upon them for nutrient supply fail to develop, are malformed, or arrest in some incomplete stage. In contrast to the prevailing view that sirenomelia arises by posterior fusion of the two developing lower limbs, these studies suggest that the single lower extremity in sirenomelia arises from failure of the lower limb bud field to be cleave.

\section{Conclusion}

Sirenomelia is a rare and lethal congenital anomaly. Termination should be offered when diagnosed antenatal, however, prevention is possible and should be the goal. Regular antenatal check-up with optimum maternal blood glucose level in pre-conception period and in first trimester should be maintained to prevent this anomaly. Controversies on its aetiopathogenesis persist. Prenatal diagnosis is possible albeit difficult by ultrasound. The associated visceral anomalies are usually incompatible with life. However surviving sirenomelic foetuses have been described with costly conservative management and mediocre results. The emphasis should be placed on prenatal diagnosis to ensure an optimal management that would consequently be less demanding both from a psychological and a health cost point of view.

\section{References}

1. Kallen,B;Castilla, E; Lancaster, P A; Mutchinnick, O; Knudsen, L B; Marrtinez-Frias, M L; Mas troiacovo, P; Robert, E. "the Cyclops and the mermaid; an epidemiological study of two types of rare malformation." J Med Genet 1992;29(1):30-5. DOI: 10.14260/jemds/1664

2. Van Keirsblick J, Cannie M, Robrechts C, de Ravel T, Dymarkowski S, Va den Bosch T, et al. First trimester diagnosis of Sirenomelia. Prenat Diagn 2006;26:68488. DOI: 10.19080/GJORM

3. Schiesser M, Holzgreve W, Lapaire O, Willi N, Luthi H, et al. Sirenomelia, the mermaid syndrome-detection in the first trimester. Prenat Diagn 2003;23:493-95. DOI: 10.16965/ijar.2014.526

4. Akbayir O, Gundorduk K, Sudolmus S, Gulkilik A, Ark C. First trimester diagnosis of Sirenomelia: a case report and review of the literature. Arch Gynecol Obstet 2008;278:589-92. DOI: 10.5455/ medscience.2016.05.8511

5. Savader SJ, Savader BL, Clark RA. Sirenomelia without Potter syndrome: MR characteristics. J

Comput Assist Tomogr 1989;13(4):689-91. DOI: 10.15226/2381-2915/3/1/00117

6. Murphy JJ, Fraser GC, Blair GK. Sirenomelia: case of the surviving mermaid. J Pediatr Surg 1992; 27(10):1265-268. DOI: https://doi.org/10.1016/00223468(92)90270-H

7. Clarke LA, Stringer DA, Fraser GC, et al.: Long term survival of an infant with sirenomelia. Am J Med Genet 1993;45(3):292-966. doi: 10.1002/ajmg.1320450303

8. Stocker JT, Heifetz SA. Sirenomelia. A morphological study of 33 cases and review of the literature. Perspect Pediatr Pathol 1987;10:7-50. DOI: [10.4103/09769668.149227]

9. Anis Fadhlaoui, Mohamed Khrouf, Soumaya Gaigi, Fethi Zhioua, Anis Chaker. The Sirenomelia Sequence: A Case History. Clin Med Insights Case Rep 2010;3:419. DOI: 10.4137/CCRep.S5347

10. Stevenson RE, Jones KL, Phelan MC, et al. Vascular steal: the pathogenic mechanism producing sirenomelia and associated defects of viscera and soft tissues. Pediatrics 1986;78:451-7. DOI: [10.4103/22494847.101699] 